\title{
Plurizentrik und DACH-Bezug bei Lehrwerken in brasilianischen Deutschstudiengängen: eine Bilanz
}

\author{
[Pluricentric and pluricultural Approaches in textbooks used in \\ German Undergraduate Courses in Brazil: an overview] \\ http://dx.doi.org/10.11606/1982-8837234124
}

\author{
Jean Paul Voerkel ${ }^{1}$ \\ Zichun Huang ${ }^{2}$
}

\begin{abstract}
This article analyzed doubts that are regularly expressed by German teachers concerning whether textbooks for teaching German as a Foreign Language (GFL) edited in Germany sufficiently represent the linguistic and cultural diversity of the entire German-speaking world. As a first step, the fundamental principles of textbook evaluation and cultural didactics in the field of GFL are discussed in this article, followed by the analysis of nine textbooks being used in German undergraduate courses of Brazilian universities in 2019 that were examined in view of an adequate representation of German speaking countries. The results suggest that the difficulties in dealing with cultural issues while teaching German are not predominantly related to the lack of suitable materials, but rather to uncertainties in handling cultural mediation. Therefore, the authors conclude with the plea for an even more conscious engagement with culture in teacher training, especially in German undergraduate courses in Brazil.
\end{abstract}

Keywords: Textbook Analysis, German as a Foreign Language (GFL), Cultural Didactics, Brazilian Universities

Resumo: O presente artigo parte da dúvida de numerosos professores de Alemão como Língua Estrangeira (ALE) sobre uma representação adequada que respeita a grande heterogeneidade linguística e cultural dos países germânicos em livros didáticos elaborados na Alemanha. Na primeira parte se situa a apresentação de algumas bases de avaliação de manuais didáticos e didática cultural sob a perspectiva da disciplina ALE. Na segunda parte, segue a análise de nove livros de ALE em uso em universidades brasileiras no ano 2019, com indicadores do "princípio DACH". Os resultados indicam que as dificuldades encontradas por professores de alemão em matéria de cultura alemã não teriam sua origem necessariamente na falta de material adequado, mas que seriam relacionadas com uma insegurança de como trabalhar conteúdo cultural em sala de aula. $\mathrm{O}$ artigo fecha com a reivindicação de integrar, ainda em escala maior, conteúdos culturais na formação de professores de alemão no Brasil.

Palavras-chave: análise de livro didático, alemão como língua estrangeira (ALE), didática cultural, universidades brasileiras

\footnotetext{
${ }^{1}$ Universidade do Estado do Rio de Janeiro, Instituto de Letras, Rua São Francisco Xavier, 524, Maracanã, Rio de Janeiro, RJ, 20550-900, Brasil. E-Mail: paul.voerkel@gmail.com. ORCID: 0000-0003-4596-1350

2 Friedrich-Schiller-Universität Jena, Ernst-Abbe-Platz 8, Jena, Thüringen, 07743, Alemanha. E-Mail: zichun.huang@uni-jena.de. ORCID: 0000-0003-1387-8835
}

(cc) BY-NC

Pandaemonium, São Paulo, v. 23, n. 41, set.-dez. 2020, p. 24-50 
Zusammenfassung: Der vorliegende Artikel greift die von Deutschlehrkräften regelmäßig geäußerten Zweifel auf, ob in Deutschland entwickelte Lehrwerke für den DaF-Unterricht die sprachliche und kulturelle Vielfalt des gesamten deutschsprachigen Raumes ausreichend darstellen. Um dieser Frage nachzugehen, werden zunächst Grundlagen der Lehrwerkanalyse und der Kulturdidaktik im Fach Deutsch als Fremdsprache erörtert. Es folgt die Darstellung der Untersuchung von neun Lehrbüchern, die 2019 in den brasilianischen Deutschstudiengängen kurstragend zum Einsatz kamen und die auf die Verwendung des DACH-Prinzips hin untersucht wurden. Die Ergebnisse legen nahe, dass die Schwierigkeiten beim Umgang mit landeskundlichen Themen im Deutschunterricht nicht vordergründig mit dem Fehlen von geeigneten Materialien zu tun haben, sondern vielmehr mit Unsicherheiten im Umgang mit der Kulturvermittlung. Es folgt deswegen abschließend ein Plädoyer für eine (noch) bewusstere Auseinandersetzung mit Kultur im Zuge der Deutschstudiengänge in Brasilien.

Stichwörter: Lehrwerkanalyse, Deutsch als Fremdsprache (DaF), Kulturelles Lernen, brasilianische Hochschulen

\section{Einleitung}

Sprachdidaktische Veröffentlichungen betonen immer wieder, dass Lehrwerke von jeher ein zentrales Element des Fremdsprachenunterrichts darstellen (vgl. VOERKEL 2015: 91, RÖSLER; SCHART 2016: 483, NIEWELER 2017a: 206, GONDAR; FERREIRA 2019: 2). Diese Tatsache ist nicht neu und gilt, im Zusammenhang mit der Sprachvermittlung, für Sprachen ganz allgemein ebenso wie für den Fachbereich Deutsch als Fremd- und Zweitsprache, der sich im deutschsprachigen Raum seit den 1970er Jahren herausgebildet und stetig weiterentwickelt hat (vgl. RÖSLER 2012: 49f., AHRENHOLZ 2017: 5f.).

Bei der Betrachtung von Lehrwerken wiederum wird schnell deutlich, wie sehr diese in ihrer Entstehungszeit verortet sind und damit die methodischen und fachlichen Diskussionen ihrer Zeit widerspiegeln (vgl. FUNK 2016: 435, NIEWELER 2017a: 206). Sichtbar wird dies insbesondere, wenn fachwissenschaftliche Diskussionen und deren Ergebnisse Eingang in die Entwicklung neuer Lehrmaterialien finden - sei es in Teilbereichen der Disziplin (Linguistik, Phonetik, Literatur, Didaktik oder Landeskunde) oder in übergreifenden Aspekten wie bspw. dem Layout und Design (vgl. RÖSLER 2012: 46, RÖSLER; SCHART 2016: 483).

Da Lehrwerke im Deutschunterricht eine so zentrale Rolle einnehmen, werden sie von den unmittelbar ins Unterrichtsgeschehen eingebundenen Personen - allen voran von den Lehrkräften - sehr genau in den Blick genommen und häufig auch kritisiert (vgl. RöSLER; SCHART 2016: 483). Ein in diesem Zusammenhang regelmäßig genanntes Thema ist die Vermittlung nicht nur sprachlicher, sondern auch kultureller Inhalte, für welche die Lehrenden sich mehr Unterstützung durch Lehrmaterialien wünschen (vgl. CHEN 2014, VOERKEL 2020). Kritisch wird dabei angemerkt, dass die in Deutschland entwickelten und vertriebenen 
Lehrwerke einerseits zu wenig auf die Ausgangssituation und (kulturellen) Vorerfahrungen der Lernenden eingehen (vgl. RÖSLER 2012: 45), andererseits aber auch die vielschichtigen Aspekte des gesamten deutschsprachigen Raumes $\left(\mathrm{DACH}^{3}\right) \mathrm{zu}$ wenig beachten und fast ausschließlich auf Deutschland orientiert seien. Es wird deswegen seit den 1990er Jahren gefordert, dass die Landeskunde, aber auch die sprachliche Darstellung die Vielfalt des deutschsprachigen Raumes besser abbilden müssen ${ }^{4}$ (vgl. RÖSLER 2012: 217f., LIMBERGER; VON MÜHLEN 2019: 338).

Die Dominanz der Bundesrepublik innerhalb der deutschsprachigen Länder ist tatsächlich nicht zu übersehen und wirkt sich auf die Darstellung Deutschlands in verschiedenen Bereichen aus - nicht nur, aber eben auch in Bezug auf die Sprache:

Zweifelsohne ist aufgrund der Größe, wirtschaftlichen Stärke und politischen Bedeutung Deutschlands die deutschländische Varietät gegenüber dem österreichischen Deutsch und dem Schweizer Hochdeutsch dominant. Das bedeutet u. a. auch, dass Teutonismen in der Regel in Österreich und der Deutschschweiz bekannter und verständlicher sind als umgekehrt Austriazismen und Helvetismen in Deutschland. Ein weiteres Merkmal dieser Asymmetrie ist, dass das deutschländische Deutsch nicht zuletzt auch von österreichischer und Schweizer Seite häufig - vor allem in Wissenschaft, Belletristik, Theater und Kunstgesang - als das korrektere, prestigehaftere und funktionell leistungsstärkere eingestuft wird. (HÄGI 2006: 275f.)

Das Zitat verdeutlicht die Relevanz des Themas: Es geht bei der Diskussion um die Verwendung des „richtigen Deutsch“ nicht nur um linguistische Formalien, sondern ganz wesentlich auch um Fragen der Identität und der gegenseitigen Einflussnahme (vgl. BoHUNOVSKY 2014: 120-124). Der Deutschunterricht steht deswegen vor der Aufgabe, eine passende Balance zwischen klaren, eindeutigen Aussagen über die DACH-Länder und der Darstellung der sprachlichen und kulturellen Vielfalt des Zielsprachenraumes zu finden.

Die Bedeutung einer vertieften Auseinandersetzung mit landeskundlichen Themen ergibt sich dabei aus der inzwischen kaum mehr in Frage gestellten engen Verknüpfung von Kultur und Sprache im Fremdsprachenunterricht (vgl. IDV 1990: 15, VoERKEL 2015: 107, KOREIK 2018: 40). Während nicht bestritten wird, dass für die Ausgestaltung des sprachlichen Anteils im Klassenzimmer eine große Breite an Materialien wünschenswert (und inzwischen auch verfügbar) ist, befindet sich die Auseinandersetzung um die passende Darstellung

\footnotetext{
${ }^{3}$ Das Akronym „DACH“ ist aus den Anfangsbuchstaben der drei deutschsprachlichen Vollzentren Deutschland, Österreich und der Schweiz gebildet. Im vorliegenden Artikel steht DACH - im Sinne von Ammon et al. (2016) - für die Voll- und Halbzentren (also auch Liechtenstein, Luxemburg, Ostbelgien und Südtirol), wobei letztere nicht mit eigenem Buchstaben (bspw. DACHL) gekennzeichnet werden (vgl. auch SHAFER; BAUMGARTNER 2019: 100, 104, 106).

${ }^{4}$ Als Beispiel dafür kann die FL-A-CH-Arbeitsgruppe genannt werden, die sich im Rahmen des USamerikanischen Deutschlehrerverbands AATG gebildet hat und in der Deutschland nominell nicht vorkommt.
} 
kultureller und landeskundlicher Themen des deutschsprachigen Raumes noch in vollem Gange. Diese Diskussion betrifft unmittelbar die Konzeptionierung und Erstellung von Lehrwerken für den Deutschunterricht sowie auch die Möglichkeiten, die Lehrkräften zur Kulturvermittlung offen stehen (vgl. LIMBERGER; VON MÜHLEN 2019: 336).

Vor diesem Hintergrund lässt sich das Interesse an einer tiefergehenden Beschäftigung mit der Kulturdidaktik verorten. Konkret stellt sich in dem Zusammenhang die Frage: Ist das bisher entwickelte Material zur Arbeit mit landeskundlichen Themen im Fremdsprachenunterricht (tendenziell) ausreichend, oder besteht nach wie vor die Notwendigkeit zur Erstellung, Neu- und Weiterentwicklung entsprechender Materialien? Dies gilt es unter Zuhilfenahme eines passenden methodischen Instrumentariums und auf der Grundlage empirischer Daten weiter zu untersuchen.

An dieser Stelle setzt der vorliegende Artikel an. Er greift dafür Aussagen von Sprachdidaktikern auf, wonach die üblicherweise im Deutschunterricht verwendeten Lehrwerke $\mathrm{zu}$ wenig auf die Plurizentrik der deutschen Sprache eingehen, die deutschsprachigen Länder weitgehend außen vorlassen und damit im Sprachunterricht zu wenig Raum für die Ausbildung eines Bewusstseins für kulturelle Differenz und Diversität lassen (vgl. JARZĄBEK 2013: 180f.; SCHWEIGER 2013: 138; LÜTGE 2016: 458). Im Folgenden geht es deswegen darum, anhand des Auftretens bestimmter Indikatoren zu überprüfen, ob dieser Vorwurf gerechtfertigt ist oder ob er vielmehr durch die Entwicklungen der letzten Jahre inzwischen als überholt angesehen werden kann.

Im Kontext der Lehrwerkanalyse liegen heutzutage verschiedene Kriterienkataloge vor, um Lehrwerke (möglichst auf Basis objektiver Indikatoren) analysieren zu können (vgl. z. B. KAST; NEUNER 1994, FUnK 2004, RÖSLER; WÜRFFEL 2014). Gleichzeitig ist bei der Auswahl von Lehrwerken für eine bestimmte Zielgruppe bis heute die „berüchtigte Daumenprobe“ (FUNK 2014: 69) üblich und es fehlen empirische Studien zur tatsächlichen Verwendung von Lehrmaterialien im Deutschunterricht. Wenn die Wirkungsforschung auf internationalem Niveau inzwischen auch das Unterrichtsgeschehen genauer betrachtet hat und viele Daten vorliegen, so bestehen doch nach wie vor große Lücken bei der theoretischen Grundlegung und der praktischen Umsetzung von Lehrwerkanalyse im Zusammenhang mit dem Deutschunterricht in der Praxis (vgl. RÖSLER; SCHART 2016: 490f., GONDAR; FERREIRA 2019: 304). Hier versucht der vorliegende Artikel anzuknüpfen, indem er zunächst kurz auf die Geschichte der Lehrwerkanalyse im Fachgebiet Deutsch als Fremd- und Zweitsprache eingeht und damit eine theoretische Grundlage setzt. Anschließend wird das methodische Vorgehen 
einer Studie beschrieben, in welcher es den Autoren darum geht, die in der brasilianischen Lehramtsausbildung verwendeten Deutsch-Lehrwerke auf ihren Bezug zu DACH-Themen bzw. dem DACH-Prinzip ${ }^{5}$ hin zu analysieren. Es folgen die Ergebnisse der Studie und abschließende Empfehlungen zur weiteren Auseinandersetzung mit landeskundlichen Fragestellungen im Fremdsprachenunterricht.

\section{Theoretischer Hintergrund}

Die folgenden Überlegungen erfolgen aus der Sicht des Faches Deutsch als Fremd- und Zweitsprache (DaF/DaZ). Zum besseren Verständnis der Argumentation des Textes wird in diesem Kapitel aus dieser Perspektive heraus - zumindest ansatzweise - auf den aktuellen Forschungsstand zur Lehrwerkanalyse, zum landeskundlichen Lernen und zu Untersuchungen zur Kulturvermittlung in Brasilien eingegangen.

\subsection{Zur Lehrwerkanalyse}

Die Lehrwerkanalyse hat in den einzelnen DaF-/DaZ-Studiengängen im deutschsprachigen Raum unterschiedliches Gewicht, wird in der Aus- und Weiterbildung von Fremdsprachenlehrkräften jedoch als zunehmend wichtig anerkannt (vgl. FUNK 2014: 78-83, FUNK 2016: 438). Zukünftige Lehrende sollten mit Lehrwerken vertraut sein, um das zur Verfügung stehende Material möglichst effektiv nutzen zu können - denn nur bei guter Kenntnis der Lehrwerke können diese auch dem Anspruch gerecht werden, die Lehrkräfte nachhaltig zu unterstützen und im Unterrichtsalltag zu entlasten (vgl. FUNK 2016: 437). Sie können vor allem dann vom Instrumentarium der Lehrwerkanalyse profitieren, wenn sie diese nicht rein hermeneutisch betreiben, sondern empirische Daten und die Komplexität des Unterrichtsgeschehens mit einbeziehen (vgl. FUnK 2004: 42, FUNK 2014: 73).

Die systematische Auseinandersetzung mit Lehrwerken ist dabei kein neues Phänomen, sondern lässt sich über mehr als 40 Jahre zurückverfolgen. Ein Anfangsimpuls war das Ende der 1970er Jahre vom Auswärtigen Amt in Auftrag gegebene „Mannheimer Gutachten“, das einen Überblick über die zur Verfügung stehenden DaF-Lehrwerke liefern sollte (vgl. FUNK 2014: 67, RÖSLER; SCHART 2016: 485). Die Ergebnisse wurden 1978/1979 veröffentlicht und

\footnotetext{
${ }^{5}$ Zur Definition des DACH-Prinzips vgl. u. a. Shafer und Baumgartner (2017: 68).

Pandaemonium, São Paulo, v. 23, n. 41, set.-dez. 2020, p. 24-50
} 
führten zu ersten tiefergehenden Diskussionen über Kriterien zur Lehrwerkanalyse im DaFBereich. Ein weiterer bedeutsamer Beitrag zur Lehrwerkanalyse war der 1994 von Kast und Neuner herausgegebene Sammelband, in dem u. a. der von Krumm erstellte „Stockholmer Katalog“ zur Lehrwerkanalyse abgedruckt ist, der als gut handhabbarer Kriterienkatalog gelten kann. Es lässt sich damit bestätigen, dass die Lehrwerkanalyse im deutschsprachigen Raum vor allem mit der Etablierung des Faches Deutsch als Fremd- und Zweitsprache und der damit verbundenen Lehr- und Lernforschung wichtige Impulse erhielt (vgl. RÖSLER 2012: 49f., NIEWELER 2017b: 208).

Dass es seit Mitte der 1990er Jahre ruhiger um die Lehrwerkanalyse geworden ist (vgl. FUNK 2004: 41, FUNK 2014: 67) sollte nicht darüber hinwegtäuschen, dass die Auseinandersetzung mit Lehrwerken im Fach sich stetig weiterentwickelt hat. Davon zeugen die Veröffentlichung von Kriterienkatalogen (z. B. FUNK 2004) und die didaktische Einbettung von Lehrmaterialien und Medien in den Unterrichtskontext (z. B. RÖSLER; WÜRFFEL 2014) ebenso wie länder- oder adressatenspezifische Studien zum Einsatz von Lehrmaterial (z. B. BOLACIO; VOERKEL; STANKE 2017). Letztere nutzen in ihrer Erhebung eine Kombination von Kriterienkatalogen, um den Gebrauch von Lehrwerken in den Lehramtsstudiengängen Brasiliens $\mathrm{zu}$ analysieren, und verbinden damit theoretische und praktische Aspekte des Lehrwerkeinsatzes für eine spezifische Zielgruppe.

\subsection{Lehrmaterialien zum landeskundlichen Lernen}

Ebenso wie die methodisch-didaktischen Leitlinien haben sich in den letzten Jahrzehnten auch die Überlegungen zum kulturellen und landeskundlichen Lernen im Fremdsprachenunterricht weiterentwickelt. Für die DaF-Landeskundedidaktik sind dabei drei große Strömungen zu nennen: die „kognitive Landeskunde“ in den 1960er und 1970er Jahren, die „kommunikative Landeskunde“ in den 1980er Jahren und der „,interkulturelle Ansatz“ seit den 1990er Jahren (vgl. VOERKEL 2015: 93-100). Alle drei Schwerpunktsetzungen, die sich z. T. überlappen und bis heute im DaF-Unterricht auftreten, haben jeweils ihre eigenen Materialien hervorgebracht.

Ergänzt - aber nicht grundsätzlich abgelöst - werden diese Landeskundeansätze seit einigen Jahren durch konstruktivistisch geprägte Vorgehensweisen einer sich neu konstituierenden kulturwissenschaftlichen Landeskunde im Fach Deutsch als Fremdsprache (vgl. VOERKEL 2015: 100f., FORNOFF 2016: 38-43), in deren Linie inzwischen auch eigene Materialien entwickelt werden (siehe z. B ALTMAYER et al. 2016). Diese Entwicklungen gehen einher mit einer zunehmenden wissenschaftlichen Fundierung plurizentrischer Ansätze des 
Deutschen (vgl. etwa DeMmig; HëGI; SCHWEIGER 2013, zur Ausformulierung des DACHPrinzips SHAFER; BAUMGARTNER 2019).

Ausgehend von den ABCD-Thesen (1990) tritt das landeskundliche Lernen immer stärker als integraler Bestandteil des Fremdsprachenlernens neben die sprachliche Komponente, was sich auch in der Ausgestaltung von Lehrwerken niederschlägt. Mit dem DACH-Konzept wird seit den 1990er Jahren versucht, die bis dato vornehmlich faktischen Informationen zu Deutschland aufzubrechen und als trinationales Landeskunde- und Fortbildungskonzept stärker kommunikativ in Lehrwerke und den Unterricht zu integrieren (vgl. BOHUNOvSKY 2014: 111f., SHAFER; BAUMGARTNER 2017: 68). Zudem wurde erkannt, dass es sinnvoll ist, in den wachsenden Märkten außerhalb Europas den deutschsprachigen Raum als integralen Teil Europas bzw. der Europäischen Union zu thematisieren ${ }^{6}$ (vgl. RöSLER 2012: 218).

Die in den letzten zehn Jahren intensivierten theoretischen Diskussionen des Fachs rund um die Bedeutung von Landeskunde und Kultur spiegeln sich auch in der Konzeption und der Ausgestaltung von DaF-/DaZ-Lehrwerken wieder, denn „... [g]anz wichtig und offener als die durch Lehrpläne vorgegebene Grammatikprogression ist die Frage, welche landeskundlich und interkulturell relevanten Inhalte und Stoffe vermittelt werden.“ (NIEWELER 2017a: 207). Die entsprechend angeregten Veränderungen und Weiterentwicklungen sind immer wieder Gegenstand von Untersuchungen (vgl. FUNK 2010: 365). Besonders relevant erscheinen Studien zu plurizentrischen und variablen Aspekten der deutschen Sprache und Kultur im brasilianischen Kontext, der sich durch eine große sprachliche Heterogenität der Mehrheitssprache Portugiesisch sowie den Sprachkontakt mit verschiedenen deutschen Varietäten auszeichnet (vgl. LIMBERGER; VON MÜHLEN 2019: 334).

\subsection{Untersuchungen zum Einsatz von Lehrmaterialien in Brasilien}

Mit der Frage, wie im Deutschunterricht mit Lehrmaterialien umgegangen wird, haben sich im brasilianischen Kontext in den letzten Jahren mehrere Untersuchungen und Publikationen beschäftigt (vgl. u. a. Chen 2014, Bolacio; Voerkel; Stanke 2017, Voerkel 2017, GONDAR; FERREIRA 2019). Aufschlussreich ist dabei, dass sich die Überlegungen dazu vor allem auf den Hochschulbereich beziehen, andere Bereiche (freie Kursangebote, Schulen,

\footnotetext{
${ }^{6}$ Ein prototypisches Beispiel dafür ist das Lehrwerk „Studio D“, in dem an verschiedenen Stellen auf „Europa“ verwiesen wird.

Pandaemonium, São Paulo, v. 23, n. 41, set.-dez. 2020, p. 24-50
} 
Nachhilfe) jedoch bisher weitgehend ausgeklammert geblieben sind. Grund dafür ist in erster Linie sicher die Verankerung der jeweiligen Akteure im Hochschulkontext und der damit verbundene Zugang zu den Materialien und Unterrichtssettings.

Neben allgemeinen Lehrwerkanalysen liegen damit bereits Informationen zum Einsatz von Unterrichtsmaterialien im Hochschulkontext vor. Was allerdings aufgrund der Breite des Themas (nachvollziehbarerweise) noch ein Desiderat darstellt, sind Untersuchungen zu bestimmten Aspekten von Lehrwerken in ihrem jeweils spezifischen Zusammenhang. So greifen beispielsweise Gondar und Ferreira (2019) in ihrem Artikel Fragen der Wortschatzarbeit, der Grammatikprogression und der Übungsformen auf, lassen jedoch Kultur und Landeskunde außen vor.

\section{Methodisches Vorgehen}

Lehrwerke können nach wie vor als die Hauptquelle für die Planung und Durchführung von Fremdsprachenunterricht angesehen werden und steuern diesen wesentlich (vgl. FUNK 2010: 364, Voerkel 2015: 91, NieWELER 2017a: 206, LiMBERGER; Von MüHLEN 2019: 337). Diese Präferenz zum aktiven Gebrauch der Materialien hat verschiedene Gründe - u. a. Vorgaben der Curricula oder der Institutionen, Zeitmangel der Lehrpersonen, Erwartungen der Lerner - und hält sich trotz der Entwicklungen im technischen Bereich weiterhin stark, denn letztendlich sind Lehrwerke ein geeignetes „Geländer“ für den fremdsprachlichen Unterricht (vgl. NIEWELER 2017a: 207). Trotzdem ist anzumerken, dass die Erforschung und v. a. die empirische Herangehensweise an Lehrwerke bisher lückenhaft ist (vgl. LIMBERGER; VoN MüHLEN 2019: 351). Lehrwerke sollen deswegen im Zentrum dieses Artikels stehen und, unter Zuhilfenahme geeigneter Instrumente, untersucht werden.

Ein wesentliches Instrument für die Lehrwerkanalyse sind Kriterienkataloge, von denen inzwischen eine ganze Reihe zur Verfügung stehen (vgl. NIEWELER 2017b: 208). Neuere Ansätze legen Wert darauf, die Kriterienkataloge nicht nur bei der Hand zu haben, sondern diese auch anzuwenden, zu adaptieren und für empirische Erhebungen zu nutzen (vgl. FUNK 2004: 41f.). Hilfreich ist in diesem Zusammenhang, mit konkreten Indikatoren zu arbeiten, um eine prozessorientierte Analyse zu gewährleisten (vgl. FUNK 2014: 74ff.).

Pandaemonium, São Paulo, v. 23, n. 41, set.-dez. 2020, p. 24-50 


\subsection{Zur Auswahl der Lehrwerke}

Aktuell bieten 17 Hochschulen in Brasilien ein eigenständiges Lehramtsstudium für Deutsch an und nutzen für die Sprachausbildung in der Regel auch Lehrwerke. Um welche Materialien es sich dabei handelt wurde in den letzten Jahren durch mehrere Untersuchungen und Beiträge dokumentiert (vgl. Andrade E Silva 2015: 228, Silveira 2015: 277f., SCHMIDT 2016, Bolacio; Voerkel; Stanke 2017: 21, Voerkel 2017: 241). Insgesamt werden in den Publikationen 17 Lehrwerke genannt $^{7}$ - allerdings ist anzumerken, dass die Angaben nicht immer übereinstimmen, was u. a. am Wechsel von Auflagen, an unvollständigen Auflistungen und an einer fehlenden Trennung zwischen Deutschstudiengängen und Unterricht an Sprachenzentren liegt. Aus diesem Grund haben die Autoren Anfang 2019 per informeller Erhebung die aktuelle Verwendung von Materialien an denjenigen Universitäten dokumentiert, die einen eigenständigen Deutschstudiengang (Graduação em Letras-Alemão) anbieten ${ }^{8}$. Die folgende Liste gibt eine entsprechende Übersicht.

Tabelle 1: Überblick über die Lehrwerke, die in brasilianischen Deutschstudiengängen verwendet werden

\begin{tabular}{|llll|}
\hline Nr. & Hochschule & Lehrwerk & Verlag \\
\hline 1 & FURB $^{9}$ & Studio d & Cornelsen \\
2 & IFPLA & Motive $^{10}$, Aspekte neu & Hueber, Klett \\
$\mid \begin{array}{ll}3 \\
4\end{array}$ & UERJ & DaF kompakt, Mittelpunkt & Klett \\
5 & UFBA & Motive & Hueber \\
6 & UFF & Studio 21 & Cornelsen \\
7 & UFMG & DaF kompakt & Klett \\
8 & UFPA & DaF kompakt & Klett \\
9 & UFPel & Optimal & Langenscheidt \\
10 & UFPR & Studio 21 & Cornelsen
\end{tabular}

\footnotetext{
${ }^{7}$ Es handelt sich dabei um die folgenden Lehrwerke (in alphabetischer Reihenfolge): Aspekte (Klett), Aussichten (Klett), Blaue Blume (Hueber), DaF kompakt (Klett), em (Hueber), Erkundungen (Schubert), Eurolingua (Cornelsen), Menschen (Hueber), Mittelpunkt (Klett), Motive (Hueber), Optimal (Langenscheidt), Passwort Deutsch (Klett), Schritte (Hueber), Studio 21 (Cornelsen), Studio d (Cornelsen), Tangram (Hueber), Ziel (Hueber). ${ }^{8}$ Trotz des Abgleichs sind möglicherweise nicht alle Angaben aktuell, da es bei der Verwendung der Lehrwerke eine starke Dynamik gibt. So wird bspw. zum Zeitpunkt der Fertigstellung des Artikels das Lehrwerk „Ziel“ an der UFRJ durch ,Sicher“ ersetzt.

${ }^{9}$ An der FURB sind 2019 keine Studierenden im Deutschstudiengang immatrikuliert, die Angaben beziehen sich deswegen auf die letzte Studienkohorte.

${ }^{10}$ Das Lehrwerk „Motive“ wird im Deutsch-Intensivkurs eingesetzt, der dem eigentlichen Studium vorgeschaltet ist und von den Studierenden durchlaufen wird, die beim Eintritt ins IFPLA noch kein B1-Niveau erreicht haben.
} 
VOERKEL, J.P.; HUANG, Z. - Plurizentrik und DACH-Bezus bei Lehrwerken

\begin{tabular}{|llll}
\hline 11 & UFRGS & DaF kompakt & Klett \\
12 & UFRJ & DaF kompakt, Ziel & Klett, Hueber \\
13 & UFSC & - kein kurstragendes Lehrwerk im Gebrauch - \\
14 & UNESP - Araraquara & Studio d, Studio 21 & Cornelsen \\
15 & UNESP - Assis & Menschen & Hueber \\
16 & UNIOESTE & Menschen & Hueber \\
17 & USP & DaF kompakt & Klett \\
\multicolumn{3}{c}{ Quelle: Eigene Erarbeitung }
\end{tabular}

Es ist klar, dass die Lehrwerke nicht die alleinige Quelle für den Deutschunterricht darstellen, sondern kontinuierlich durch verschiedene Zusatzmaterialien ergänzt werden (vgl. u. a. FUNK 2016: 436). Gleichzeitig zeigt die Praxis, dass die Lehrwerke als „Ankermedium“ (FUNK 2016: 440) die zentrale Stütze für den Unterricht darstellen und sich deswegen im Unterrichtsalltag unentbehrlich machen (vgl. FUnK 2016: 438, BOLACIO; VOERKEL; STANKE 2017: 22, GONDAR; FERREIRA 2019: 310f.). Dies ist insbesondere an den öffentlichen Institutionen in Brasilien der Fall, in denen ein häufig eingeschränkter Zugang zum Internet und zur Nutzung neuer Medien den Fokus nach wie vor auf das Lehrbuch lenkt (vgl. GoNDAR; FERREIRA 2019: 323). Anzumerken ist zudem, dass in der Auflistung die Institutionen fehlen, die Deutsch zwar anbieten (sei es als Bachelor-Studiengang oder in freien Kursen), aber keinen eigenständigen Deutschstudiengang betreiben ${ }^{11}$. Die hierfür notwendigen Zahlen wurden Mitte 2019 im Zuge der Deutschlernererhebung des „Netzwerks Deutsch“ erhoben und sind zum gegenwärtigen Zeitpunkt noch nicht veröffentlicht.

$\mathrm{Da}$ es in dem Artikel um die Lehrwerke geht, mit denen die Studierenden der Deutschstudiengänge (Graduação em Letras-Alemão) in Brasilien arbeiten, wurden die folgenden Lehrwerke für die Analyse ausgewählt:

Tabelle 2: Für die Analyse ausgewählte Lehrwerke

\begin{tabular}{|cllc|}
\hline Nr. & Lehrwerk & Verlag & Nennungen \\
\hline 1 & Aspekte & Klett & 1 \\
2 & DaF kompakt & Klett & 7 \\
3 & Menschen & Hueber & 3 \\
4 & Mittelpunkt & Klett & 1
\end{tabular}

\footnotetext{
${ }^{11}$ Dies ist der Fall bspw. bei den Universitäten UFPB, UFRN und UNICAMP.

Pandaemonium, São Paulo, v. 23, n. 41, set.-dez. 2020, p. 24-50
} 
VOERKEL, J.P.; HUANG, Z. - Plurizentrik und DACH-Bezus bei Lehrwerken

\begin{tabular}{|llll}
5 & Motive & Hueber & 2 \\
6 & Optimal & Langenscheidt & 1 \\
7 & Studio 21 & Cornelsen & 3 \\
8 & Studio d & Cornelsen & 2 \\
9 & Ziel & Hueber & 1
\end{tabular} \mid

Quelle: eigene Darstellung

Die Tabelle zeigt einige Informationen, die es sich näher zu betrachten lohnt. Bis auf eine Ausnahme nutzen alle Hochschulen für den Sprachunterricht kurstragende Lehrwerke, die in Deutschland entwickelt wurden (vgl. GONDAR; FERREIRA 2019: 309). Die Liste macht zudem deutlich, dass die Lehrwerke ausnahmslos aus den drei großen deutschen Verlagen mit dem breitesten DaF-Angebot kommen (Cornelsen, Hueber, Klett). Außerdem ist es anzumerken, dass es bei einigen Hochschulen gerade einen Übergang gibt - so z. B. von „Studio d“ zu „Studio 21“ (z. B. bei UNESP Araraquara) oder von „DaF kompakt“ zu „DaF kompakt neu“ (z. B. an der UERJ).

Ausgehend von der tatsächlichen Verwendung der Lehrwerke und den nachfolgenden Beobachtungen wurden letztendlich die folgenden Lehrbücher für die Analyse genutzt:

Tabelle 3: Analysierte Lehrbücher

\begin{tabular}{|c|c|c|c|c|c|c|}
\hline Nr. & Lehrwerk & Band & Niveau & Jahr & Seitenzahl & Kapitel / Module \\
\hline \multirow[t]{2}{*}{1} & Aspekte & 1 & B2.1 & 2008 & 80 & $1-5(5)$ \\
\hline & & 2 & B 2.2 & 2008 & 80 & $6-10(5)$ \\
\hline \multirow[t]{3}{*}{2} & DaF kompakt neu & 1 & A1 & 2016 & 64 & $1-8(8)$ \\
\hline & & 2 & A2 & 2017 & 80 & 9-18 (10) \\
\hline & & 3 & B1 & 2018 & 96 & $19-30(12)$ \\
\hline \multirow[t]{3}{*}{3} & Menschen & 1 & A1 & 2012 & 128 & $1-8(8)$ \\
\hline & & 2 & $\mathrm{~A} 2$ & 2013 & 128 & $1-8(8)$ \\
\hline & & 3 & B1 & 2015 & 144 & $1-8(8)$ \\
\hline \multirow[t]{2}{*}{4} & Mittelpunkt & 2 & B2 & 2007 & 144 & $1-12(12)$ \\
\hline & & 3 & $\mathrm{C} 1.1$ & 2008 & 72 & $1-6(6)$ \\
\hline \multirow[t]{3}{*}{5} & Motive & 1 & A1 & 2015 & 68 & $1-8(8)$ \\
\hline & & 2 & A2 & 2015 & 80 & $9-18(10)$ \\
\hline & & 3 & B1 & 2016 & 96 & 19-30 (12) \\
\hline 6 & Optimal & 3 & B1 & 2006 & 114 & 1-Ausklang (15) \\
\hline
\end{tabular}


VOERKEL, J.P. $;$ HUANG, Z. - Plurizentrik und DACH-Bezus bei Lehrwerken

\begin{tabular}{|c|c|c|c|c|c|c|}
\hline \multirow[t]{3}{*}{7} & Studio 21 & 3 & A 2.1 & 2014 & 116 & 1-Station 2 (8) \\
\hline & & 4 & A 2.2 & 2015 & 112 & 7-Station $4(8)$ \\
\hline & & 5 & B 1.2 & 2015 & 104 & 1-Station 1 (6) \\
\hline \multirow[t]{2}{*}{8} & Studio d & 1 & A1 & 2005 & 200 & 1-Station $3(15)$ \\
\hline & & 2 & $\mathrm{~A} 2$ & 2006 & 206 & 1-Station 4 (16) \\
\hline \multirow[t]{2}{*}{9} & Ziel & 1 & B2.1 & 2008 & 112 & $1-8(8)$ \\
\hline & & 2 & B 2.2 & 2009 & 112 & $9-16(8)$ \\
\hline \multicolumn{2}{|c|}{ Gesamt: 9 Lehrwerke } & 21 & A1.1 - C1.1 & $2005-18$ & 2.336 & 196 \\
\hline
\end{tabular}

Quelle: eigene Darstellung

Im Rahmen der Analyse der neun Lehrwerke wurden 21 (Teil-)Bände der Niveaus A1.1 bis C1.1 untersucht, wobei die Gesamtmenge 2.336 Seiten und 196 Kapitel umfasst. Die ausgewählten Lehrwerke erschienen in den Jahren zwischen 2005 und 2018 und richten sich an unterschiedliche Zielniveaus. Wichtig ist zu erwähnen, dass die Sichtung nur die Lehrbücher umfasste und (v. a. aus Gründen der Verfügbarkeit) nicht alle Teilbände vertreten sind. Im Wesentlichen handelt es sich jedoch um die Bände, die im Deutschunterricht an den Universitäten tatsächlich zum Einsatz kommen - insofern kann trotz der erwähnten Einschränkungen von einer hohen Repräsentativität gesprochen werden.

\subsection{Zur methodischen Grundleguns}

Die Untersuchung reiht sich in Überlegungen zum DACH-Prinzip ein und fragt danach, in welchem Umfang dieses in den analysierten Lehrwerken in Erscheinung tritt. Das DACHPrinzip als solches postuliert eine angemessene Anerkennung der sprachlichen und kulturellen Vielfalt des deutschsprachigen Raumes, die im Folgenden näher beschrieben werden.

Die sprachliche Seite bezieht sich auf die Plurizentrik des Deutschen. Plurizentrik ist gegeben, wenn eine Sprache in mehreren Ländern bzw. Regionen die offizielle, anerkannte Landessprache ist und mehrere Varianten beinhaltet - was für die deutsche Sprache zweifelsohne zutrifft (vgl. MUHR 2000, JARZĄMBEK 2013: 173, BOHUNOvSKY 2014: 111, LIMBERGER; VON MÜHLEN 2019: 335f.). Um zu erfassen, ob die Plurizentrik des Deutschen in den Lehrwerken aufgegriffen wird, kann als Indikator gelten, in welchem Umfang auf die linguistischen Eigenheiten der deutschländischen, österreichischen und schweizerischen 
Varietäten eingegangen wird ${ }^{12}$. Deutlich wird dies v. a. über die Nennung von Teutonismen, Austriazismen und Helvetismen (vgl. BOHUNOvSKY 2014: 115).

Bei kulturell-landeskundlichen Inhalten ist ein möglicher Indikator für die Berücksichtigung des DACH-Prinzips zunächst eine ausgewogene Darstellung von Themen und Inhalten aus den DACH-Ländern und ihre jeweilige Evozierung. In Hinblick auf den praktischen Einsatz kann an dieser Stelle mit der Unterscheidung zwischen ,impliziter“ vs. „expliziter“ Verwendung gearbeitet werden (vgl. SHAFER; BAUMGARTNER 2019: 107). Unter einer ,impliziten Umsetzung“ ist dabei „ein selbstverständlicher, unmarkierter Einsatz von Texten, Inhalten, Varietäten, Lehrwerkfiguren Autor/inn/en etc. aus dem gesamten amtlich deutschprachigen Raum gemeint“" (SHAFER; BAUMGARTNER 2017: 69), ohne eine bestimmte Herkunft oder Aussprache bewusst zu thematisieren oder zu vergleichen. Letzteres ist bei der „expliziten Umsetzung“ der Fall, denn diese ,zeichnet sich durch eine bewusste Reflexion über die sprachliche oder kulturelle Pluralität der Unterrichtsgegenstands (,Deutsch“ bzw. „DACHRaum“) aus“ (SHAFER \& BAUMGARTNER 2017: 69) und orientiert sich an der (diskursiven) Konstruktion bestimmter kultureller Ordnungskategorien.

Ziel der Analyse der in Brasilien gebräuchlichen Lehrwerke war es also einerseits, die Berücksichtigung der deutschsprachigen Länder implizit und explizit offen zu legen, und andererseits das in plurizentrischen Ansätzen ebenfalls postulierte Verbindende zu erfassen (vgl. HÄGI 2006: 275).

\subsection{Zur Durchführung der Analyse}

Lehrmaterialien umfassen heutzutage nicht nur das „Lehrbuch“, sondern einen ganzen Lehrwerksverbund, der aus verschiedenen Komponenten besteht (vgl. FunK 2016: 436, NIEWELER 2017a: 206, GONDAR; FERREIRA 2019: 303). Der Pragmatik halber bezieht sich die vorliegende Untersuchung jedoch ausschließlich auf die Lehrbücher, die im Unterricht nach wie vor eine zentrale Stütze darstellen (vgl. NIEWELER 2017a: 207, LIMBERGER; VON MÜHLEN 2019: 351). Dies entspricht - trotz der grundsätzlich wünschenswerten Gesamtsicht - der gängigen Praxis der Lehrwerkanalyse, die Arbeitsbücher, Lehrerhandreichungen oder Hörtexte weit weniger häufig zum Untersuchungsgegenstand macht (vgl. FunK 2016: 439). Zudem

\footnotetext{
${ }^{12}$ Die Betrachtungen erstrecken sich in der Regel (und auch im vorliegenden Artikel) nur auf Deutschland, Österreich und die Schweiz, da als Zentren einer plurizentrischen Sprache nur diejenigen Länder oder Regionen gelten, die eigene standardsprachliche Besonderheiten ausgebildet haben (vgl. AMMON 2016).

Pandaemonium, São Paulo, v. 23, n. 41, set.-dez. 2020, p. 24-50
} 
konnten aus Gründen der Verfügbarkeit und des Umfangs der Studie nicht alle Bände der genannten Lehrwerke durchgesehen werden - bei den eingesehenen Büchern handelt es sich jedoch in der Regel um die Bände, die in den jeweiligen Institutionen eingesetzt werden. Insofern entspricht die Auswahl zwar nicht der Gesamtmenge des potenziell zur Verfügung stehenden Materials, wohl aber dem Einsatz in Brasilien. Unabhängig davon stellen die über 2.000 gesichteten Seiten eine repräsentative Größe dar und erlauben es, bestimmte Tendenzen abzulesen.

Zur Analyse und Dokumentation entschieden sich die Verfasser dafür, alle Lehrwerke mithilfe einer Tabelle zu sammeln und darzustellen, um die Daten für die Analyse in gleichwertiger Form vorliegen zu haben. Die zugrunde liegende Tabelle hatte dabei die folgende Form:

Abbildung 1: Raster für die Zählung der DACHL-Beispiele

\begin{tabular}{|c|c|c|c|c|c|c|c|c|c|}
\hline \multicolumn{10}{|c|}{ Name des Lehrwerks, Niveaustufe nach GeR, Teilband } \\
\hline \multicolumn{10}{|c|}{ Jahr des Erscheinens, Verlag, Zielgruppe } \\
\hline \multicolumn{10}{|c|}{ Seiten- und Kapitelzahl } \\
\hline \multirow{2}{*}{$\begin{array}{l}\text { Nr. der } \\
\text { Lektion }\end{array}$} & \multicolumn{2}{|c|}{ Deutschland } & \multicolumn{2}{|c|}{ Österreich } & \multicolumn{2}{|l|}{ Schweiz } & \multicolumn{2}{|c|}{ Liechtenstein } & $\begin{array}{l}\text { Bsp. } \\
\text { DACH }\end{array}$ \\
\hline & implizit & explizit & implizit & explizit & implizit & explizit & implizit & explizit & \\
\hline
\end{tabular}

Quelle: eigene Erarbeitung

Die Analyse der Lehrbücher erfolgte in mehren Stufen. Zunächst wurde überprüft, ob sich in den einzelnen Lektionen Verweise auf Deutschland, Österreich, die Schweiz oder Liechtenstein finden. War dies gegeben, wurde geschaut, ob die Verweise implizit oder explizit erfolgten. Darüber hinaus wurde markiert, wenn in den einzelnen Lektionen ein binnendifferenziertes Vorgehen vorgeschlagen, also ein Vergleich zwischen den deutschsprachigen Ländern angestellt wird.

Es ist an dieser Stelle anzumerken, dass bei der Analyse insbesondere die Unterscheidung zwischen ,impliziter“ und „expliziter“ Nennung nicht immer einfach war und durchaus auch Raum zur Interpretation lässt. So stellt sich z. B. die Frage, wie die Zählung erfolgen sollte, wenn von einem „Hotel in Österreich“ die Rede ist - handelt es sich, wie z. B. in Station 4 des Lehrbuchs Studio D A2, um eine explizite oder eine implizite Nennung? Die Verfasser entschieden sich dafür, die Informationen als ,implizit“ zu werten, wenn ein Beruf 
oder eine Tätigkeit im Vordergrund steht, aber als „explizit“, wenn es um einen konkreten Ort (in dem Fall die Ferienregion Tirol) geht. Um Abweichungen zu vermeiden, wurde nach dem „Vieraugenprinzip“ gearbeitet, das heißt, die gesamten Zählungen wurden gemeinsam durchgeführt und unklare Fälle zunächst angesprochen und genauer analysiert, bevor sie in die jeweilige Tabelle eingetragen wurden.

Ein weiterer erwähnenswerter Aspekt bezieht sich auf die verschiedenen verfügbaren Ausgaben der Lehrbücher - diese liegen, je nach Lehrwerk, als Gesamt- oder Teilbände vor. Aus Gründen der Übersicht und Vergleichbarkeit wurden die Teilbände, wenn mit diesen gearbeitet wurde, zusammengefasst und als ein Band gezählt.

\subsection{Zur Darstellung der Ergebnisse}

Nach der Erfassung der Daten wurde geschaut, in welchem Verhältnis das Auftreten der Nennungen zur Anzahl der Kapitel und der Seitenzahl steht. Dazu wurde die Menge der Nennungen addiert und mit der Anzahl der Kapitel verrechnet. Dementsprechend wurden die Lehrbücher wie im folgenden Beispiel dargestellt:

Abbildung 2: Raster für die Zählung der DACH-Beispiele (ausgefüllt, Beispiel)

\begin{tabular}{|c|c|c|c|c|c|c|c|c|c|}
\hline \multicolumn{10}{|c|}{ DaF kompakt neu ${ }^{13}$, A1 (Lehrbuch) } \\
\hline \multicolumn{10}{|c|}{ Klett, $2016 \rightarrow$ LW für Studierende + Berufstätige } \\
\hline \multicolumn{10}{|c|}{ Seiten- und Kapitelzahl } \\
\hline \multirow{2}{*}{$\begin{array}{l}\text { Nr. der } \\
\text { Lektion }\end{array}$} & \multicolumn{2}{|c|}{ Deutschland } & \multicolumn{2}{|c|}{ Österreich } & \multicolumn{2}{|c|}{ Schweiz } & \multicolumn{2}{|c|}{ Liechtenstein } & \multirow{2}{*}{$\begin{array}{c}\text { Bsp. } \\
\text { DACH }\end{array}$} \\
\hline & implizit & explizit & implizit & Explizit & implizit & explizit & implizit & explizit & \\
\hline 1 & & $\mathrm{x}$ & & $\mathrm{X}$ & & $\mathrm{x}$ & & $\mathrm{x}$ & $\mathrm{x}$ \\
\hline 2 & $\mathrm{X}$ & $\mathrm{X}$ & $\mathrm{X}$ & $\mathrm{X}$ & & & & & $\mathrm{X}$ \\
\hline 3 & & $\mathrm{x}$ & & $\mathrm{X}$ & & $\mathrm{x}$ & & & $\mathrm{x}$ \\
\hline 4 & $\mathrm{X}$ & & & & & & & & $\mathrm{x}$ \\
\hline 5 & $\mathrm{x}$ & & & & & & & & \\
\hline 6 & $x$ & & & & & & & & \\
\hline
\end{tabular}

\footnotetext{
${ }^{13}$ Dass sich das Beispiel auf das Lehrbuch der Serie „DaF kompakt“ bezieht ist kein Zufall, denn dieses wird an brasilianischen Hochschulen mit Abstand am meisten verwendet. Gründe, warum sich das Lehrwerk an den Universitäten durchgesetzt hat, finden sich u. a. bei Gondar und Ferreira (2019: 309) oder Marques-Schäfer, Bolacio und Stanke (2016: 572f.).
} 


\begin{tabular}{|c|c|c|c|c|c|c|c|c|c|}
\hline 7 & $\mathrm{x}$ & & $\mathrm{x}$ & & & & & & $\mathrm{x}$ \\
\hline 8 & $\mathrm{x}$ & & & & & $\mathrm{x}$ & & & $\mathrm{x}$ \\
\hline gesamt & $\mathbf{7}$ & $\mathbf{3}$ & $\mathbf{2}$ & $\mathbf{3}$ & $\mathbf{0}$ & $\mathbf{3}$ & $\mathbf{0}$ & $\mathbf{1}$ & $\mathbf{6}$ \\
\hline
\end{tabular}

Quelle: eigene Erarbeitung

Zweifelsohne ist dieses Vorgehen nicht immer ganz trennscharf, denn die Kapitel sind unterschiedlich lang, und nicht immer wurden alle Bände des gesamten Lehrwerks analysiert. Es ist den Verfassern bewusst, dass die Zahlen deswegen möglicherweise differieren können. Durch die Verwendung des Vieraugenprinzips, den Bezug auf alle genutzten Lehrwerke und die vorliegende Datenmenge kann aber davon ausgegangen werden, dass die Tendenzen, die mit den Ergebnissen sichtbar werden, als valide einzustufen sind.

\section{Ergebnisse}

Mit der Analyse der Lehrwerke wurde eine große Menge an Daten generiert. Um diese auf eine übersichtliche Weise darzustellen, fassten die Autoren die Werte der einzelnen Lehrwerke in einer Tabelle zusammen, in der die Anzahl der analysierten Lektionen und das Auftreten der Bezüge zu Deutschland, Österreich, der Schweiz, Liechtenstein und dem DACH-Prinzip ablesbar ist. Damit zeigt sich folgendes Panorama:

Tabelle 4: Ergebnisse der analysierte Lehrbücher

\begin{tabular}{|c|c|c|c|c|c|c|c|c|c|c|}
\hline \multirow[t]{2}{*}{ Lehrwerk } & \multirow{2}{*}{$\begin{array}{l}\text { Anzahl } \\
\text { d. Lekt. }\end{array}$} & \multicolumn{2}{|c|}{ Deutschland } & \multicolumn{2}{|c|}{ Österreich } & \multicolumn{2}{|c|}{ Schweiz } & \multicolumn{2}{|c|}{ Liechtenstein } & \multirow{2}{*}{$\begin{array}{c}\text { Bsp. } \\
\text { DACH }\end{array}$} \\
\hline & & impl. & expl. & impl. & expl. & impl. & expl. & impl. & expl. & \\
\hline \multirow{2}{*}{ Aspekte } & 10 & 10 & 5 & 2 & 2 & 3 & 0 & 0 & 0 & 2 \\
\hline & $100 \%$ & $100 \%$ & $50 \%$ & $20 \%$ & $20 \%$ & $30 \%$ & $0 \%$ & $0 \%$ & $0 \%$ & $20 \%$ \\
\hline \multirow{2}{*}{$\begin{array}{c}\text { DaF } \\
\text { kompakt neu }\end{array}$} & 30 & 12 & 17 & 5 & 9 & 3 & 7 & 0 & 4 & 17 \\
\hline & $100 \%$ & $40 \%$ & $57 \%$ & $17 \%$ & $30 \%$ & $10 \%$ & $23 \%$ & $0 \%$ & $13 \%$ & $57 \%$ \\
\hline \multirow[t]{2}{*}{ Menschen } & 24 & 17 & 17 & 10 & 3 & 5 & 4 & 1 & 0 & 7 \\
\hline & $100 \%$ & $71 \%$ & $71 \%$ & $42 \%$ & $13 \%$ & $21 \%$ & $17 \%$ & $4 \%$ & $0 \%$ & $29 \%$ \\
\hline \multirow[t]{2}{*}{ Mittelpunkt } & 18 & 15 & 7 & 3 & 0 & 5 & 0 & 0 & 0 & 0 \\
\hline & $100 \%$ & $83 \%$ & $39 \%$ & $17 \%$ & $0 \%$ & $28 \%$ & $0 \%$ & $0 \%$ & $0 \%$ & $0 \%$ \\
\hline Motive & 30 & 24 & 9 & 7 & 6 & 8 & 7 & 0 & 0 & 4 \\
\hline
\end{tabular}


VOERKEL, J.P.; HUANG, Z. - Plurizentrik und DACH-Bezus bei Lehrwerken

\begin{tabular}{|c|c|c|c|c|c|c|c|c|c|c|}
\hline & $100 \%$ & $80 \%$ & $30 \%$ & $23 \%$ & $20 \%$ & $27 \%$ & $23 \%$ & $0 \%$ & $0 \%$ & $13 \%$ \\
\hline \multirow{3}{*}{ Optimal } & 15 & 12 & 4 & 4 & 2 & 7 & 3 & 0 & 0 & 3 \\
\cline { 2 - 12 } & $100 \%$ & $80 \%$ & $27 \%$ & $27 \%$ & $13 \%$ & $47 \%$ & $20 \%$ & $0 \%$ & $0 \%$ & $20 \%$ \\
\hline \multirow{3}{*}{ Studio D } & 31 & 26 & 26 & 7 & 5 & 8 & 5 & 0 & 0 & 12 \\
\cline { 2 - 12 } & $100 \%$ & $84 \%$ & $84 \%$ & $22 \%$ & $16 \%$ & $26 \%$ & $16 \%$ & $0 \%$ & $0 \%$ & $39 \%$ \\
\hline \multirow{2}{*}{ Studio 21 } & 22 & 20 & 18 & 6 & 3 & 6 & 6 & 0 & 0 & 9 \\
\cline { 2 - 12 } & $100 \%$ & $91 \%$ & $82 \%$ & $27 \%$ & $14 \%$ & $27 \%$ & $27 \%$ & $0 \%$ & $0 \%$ & $41 \%$ \\
\hline \multirow{2}{*}{ Ziel } & 16 & 13 & 2 & 3 & 0 & 4 & 0 & 0 & 0 & 3 \\
\cline { 2 - 11 } & $100 \%$ & $81 \%$ & $13 \%$ & $19 \%$ & $0 \%$ & $25 \%$ & $0 \%$ & $0 \%$ & $0 \%$ & $19 \%$ \\
\hline
\end{tabular}

Quelle: eigene Darstellung

Es mag überraschen, dass sich die prozentuale Verteilung der Darstellung zwischen den Lehrwerken so deutlich unterscheidet. Eine mögliche Erklärung ist bei genauerem Hinsehen jedoch einfach zu finden: Manche Lehrwerke arbeiten eher mit Beispielen und Bildern, die abstrakt sind und keinen konkreten Ortsbezug aufweisen, in anderen Lehrwerken werden jedoch real existierende Beispiele und Bilder verwendet, die damit auch eine räumlichgeografische Verortung der Situation zulassen und somit - entweder implizit oder explizit - für einen bestimmten geografischen, sprachlichen und kulturellen Raum stehen. Wenn also die Ergebnisse aller untersuchter Lehrwerke zusammengefasst werden, lässt sich anhand einer Tabelle folgendes Bild erkennen:

Tabelle 5: Ergebnisse der analysierten Lehrbücher - Zusammenfassung

\begin{tabular}{|c|c|c|c|c|c|c|c|c|c|c|}
\hline Lehrwerk & \multirow{2}{*}{$\begin{array}{l}\text { Anzahl } \\
\text { d. Lekt. }\end{array}$} & \multicolumn{2}{|c|}{ Deutschland } & \multicolumn{2}{|c|}{ Österreich } & \multicolumn{2}{|c|}{ Schweiz } & Liechtenstein & $\begin{array}{c}\text { Bsp. } \\
\text { DACH }\end{array}$ \\
\cline { 2 - 11 } & & impl. & expl. & impl. & expl. & impl. & expl. & impl. & expl. & \\
\hline gesamt & 196 & 149 & 105 & 47 & 30 & 49 & 32 & 1 & 4 & 57 \\
\cline { 2 - 12 } & $100 \%$ & $\mathbf{7 6 \%}$ & $\mathbf{5 4 \%}$ & $\mathbf{2 4 \%}$ & $\mathbf{1 5 \%}$ & $\mathbf{2 5 \%}$ & $\mathbf{1 6 \%}$ & $\mathbf{0 , 5 \%}$ & $\mathbf{2 \%}$ & $\mathbf{2 9 \%}$ \\
\hline
\end{tabular}

Quelle: eigene Darstellung

Bei den in Brasilien für die „Lehramtsausbildung Deutsch“ verwendeten Lehrwerken wird Deutschland in drei von vier Kapiteln implizit und in etwas mehr als der Hälfte explizit thematisiert. Österreich und die Schweiz haben fast identische Werte: in etwa einem Viertel der Kapitel erfolgt es eine implizite Erwähnung, eine explizite in jedem siebten Kapitel. Liechtenstein wird sehr viel weniger thematisiert (in lediglich zwei Lehrwerken). Beispiele für Gemeinsamkeiten des DACH-Raums treten in über einem Viertel der untersuchten Kapitel auf. 
Anhand der Ergebnisse wird zunächst erkennbar, dass die Nennung von Deutschland in den Lehrwerken klar überwiegt. Österreich und die Schweiz kommen deutlich seltener vor, Liechtenstein fast gar nicht. Haben also die Kritiker recht, wenn sie sagen, dass DACH in den in Deutschland produzierten Lehrwerken nicht ausreichend zur Geltung kommt? Und wird hiermit ein Trend verstärkt, dem zufolge Deutschlerner häufig denken, dass es ausreicht und am sinnvollsten ist, sich auf Deutschland und die deutschländische Varietät zu beschränken (vgl. BoHUNOVSKY 2014: 113)? Die Antwort auf diese Fragen hängt stark von der Sichtweise ab, wobei sowohl die Motivation der Deutschlernenden als auch sprachenpolitische und didaktische Aspekte zu bedenken sind. Beide Dimensionen sollen im Folgenden näher betrachtet werden.

Schaut man sich allein die Zahlen an, so ist klar, dass Deutschland an Größe und Einwohnerzahl die beiden südlichen Nachbarländer Österreich und die Schweiz deutlich übertrifft. Im Jahr 2018 hatte Deutschland knapp 83 Millionen Einwohner ${ }^{14}$, Österreich knapp 9 Millionen ${ }^{15}$ und die Schweiz ca. 8,5 Millionen ${ }^{16}$ - die Bevölkerung Deutschlands umfasste also fast zehnmal mehr Einwohner als die der anderen beiden DACH-Länder.

Lohnenswert ist bei der Frage nach Größe und Präsenz ein zweiter Blick, und zwar der auf die Hochschulen: dieser ist nicht ganz unwesentlich, wenn man bedenkt, dass etwa zehn Prozent der Deutschlerner in Brasilien Kurse an Universitäten eingeschrieben sind und auch die Deutschkurse des Goethe-Instituts zu ca. $80 \%$ von Personen mit Studienwunsch im deutschsprachigen Ausland besucht werden ${ }^{17}$ (vgl. AuswärTiGeS AMT 2015: 9, 27). Die sprachliche (und kulturelle) Vorbereitung auf einen Studien- oder Forschungsaufenthalt in Zentraleuropa ist in Brasilien als Motiv zum Deutschlernen also ernst zu nehmen.

Bei der Anzahl der Hochschulen zeigt sich denn auch eine ähnliche - wenn auch nicht ganz so deutliche - Tendenz in Größe und Anzahl: Während es in Deutschland ca. 400

\footnotetext{
${ }^{14}$ Statistisches Bundesamt, INSEE, ONS Vereinigtes Königreich. Abrufbar unter: https://ec.europa.eu/eurostat/ en/web/population-demography-migration-projections/statistics-illustrated [Zugriff: 24.03.2019]

${ }^{15}$ Eurostat, Vereinte Nationen. Abrufbar unter: https://ec.europa.eu/eurostat/en/web/population-demographymigration-projections/statistics-illustrated [Zugriff: 24.03.2019]

16 Eurostat. Abrufbar unter: https://ec.europa.eu/eurostat/en/web/population-demography-migrationprojections/statistics-illustrated [Zugriff: 24.03.2019]

${ }^{17}$ Angabe des Goethe-Instituts beim Treffen des „Netzwerks Deutsch“ im November 2019 in Belo Horizonte.

Pandaemonium, São Paulo, v. 23, n. 41, set.-dez. 2020, p. 24-50
} 
Hochschulen gibt ${ }^{18}$, verfügt Österreich über $70^{19}$ und die Schweiz über $40^{20}$. An den universitären Einrichtungen studierten insgesamt ca. 2,8 Millionen (Deutschland), 0,4 Millionen (Österreich) bzw. 0,25 Millionen Menschen (Schweiz). In Hinblick auf die Anzahl der ausländischen Studierenden ist die Reihenfolge die gleiche (Deutschland mit knapp 360.000 ausländischen Studierenden, Österreich mit knapp 100.000 und die Schweiz mit gut 61.000). Wenn man sich jedoch die Prozentzahlen anschaut, führt Österreich mit einem Anteil von 27\% ausländischen Studierenden, gefolgt von der Schweiz, mit 25\% $\%^{21}$, und somit immer noch etwa doppelt so viel wie in Deutschland, wo der Anteil an ausländischen Studierenden nur 13\% beträgt ${ }^{22}$. Während also die Prozentzahlen in Deutschland und der Schweiz auch etwa denen der gesamten Zahl der Ausländer entsprechen, liegt dieser Wert in Österreich deutlich höher ${ }^{23}$. Die Anzahl der Hochschulen und die Forschungsstärke spiegelt sich übrigens auch in den internationalen Rankings wider: Laut dem QS-Ranking, das weltweit am meisten rezipiert wird $^{24}$, liegen von den Top-500-Universitäten weltweit 29 in Deutschland, acht in der Schweiz (davon fünf in der Deutsch-Schweiz) und vier in Österreich; das durchaus europa-freundlichere Times-Higher-Education-Ranking ${ }^{25}$ listet unter den Top-500-Universitäten weltweit 40 in Deutschland, zehn in der Schweiz (davon fünf in der Deutsch-Schweiz) und sieben in Österreich. Unter diesem Blickwinkel erscheint es zunächst legitim, wenn Deutschland in den allgemeinsprachlichen Lehrwerken deutlich häufiger in Erscheinung tritt als die anderen beiden DACH-Länder.

Doch gilt es hier umgehend zu relativieren: nicht alle Studieninteressenten orientieren sich automatisch nach Deutschland. Und noch deutlich relevanter: Zielgruppe der vorliegenden Untersuchung sind zunächst einmal die ca. 1.300 Studierenden des Lehramtsstudiums Deutsch in Brasilien (vgl. VOERKEL 2017: 263). Für sie - als zukünftige Mittler und Multiplikatoren für

\footnotetext{
18 Statista. Abrufbar unter: https://de.statista.com/statistik/daten/studie/247238/umfrage/hochschulen-indeutschland-nach-hochschulart/ [Zugriff: 24.03.2019]

19 Bundesministerium für Bildung, Wissenschaft und Forschung. Abrufbar unter: https://bmbwf.gv.at/ wissenschaft-hochschulen/ [Zugriff: 24.03.2019]

${ }^{20}$ Staatssekretariat für Bildung, Forschung und Innovation. Abrufbar unter: https://www.sbfi.admin.ch/dam/sbfi/ de/dokumente/2017/04/factsheet-hs.pdf.download.pdf/Factsheet_Hochschulen_CH_de.pdf+\&cd=1\&hl=de\&ct= clnk\&gl=br [Zugriff: 05.11.2019]

${ }^{21}$ Staatssekretariat für Bildung, Forschung und Innovation. Abrufbar unter: https://www.sbfi.admin.ch/dam/sbfi/ de/dokumente/2017/04/factsheet-hs.pdf.download.pdf/Factsheet_Hochschulen_CH_de.pdf $+\& c d=1 \&$ hl=de\&ct= clnk\&gl=br [Zugriff: 05.11.2019]

${ }^{22}$ Wissenschaft weltoffen. Abrufbar unter: http://www.wissenschaft-weltoffen.de [Zugriff: 24.03.2019]

${ }^{23}$ Warum dies so ist kann an dieser Stelle aus Platzgründen nicht weiter thematisiert werden.

${ }^{24} \mathrm{Vgl}$. die Informationen aus dem QS-Ranking 2020, abrufbar unter:

https://www.topuniversities.com/university-rankings/world-university-rankings/2020 [Zugriff: 30.01.2020]

${ }^{25}$ Daten abrufbar unter: https://www.timeshighereducation.com/world-university-rankings [Zugriff: 30.01.2020]
}

Pandaemonium, São Paulo, v. 23, n. 41, set.-dez. 2020, p. 24-50 
deutsche Sprache und Kultur - ist es ohne jeden Zweifel relevant, etwas über die Vielfalt der deutschsprachigen Länder zu erfahren, und eben nicht nur über Deutschland. Schließlich ist zu erwarten, dass sie - egal welche Zielgruppe sie später einmal unterrichten - über kurz oder lang mit Fragen von Kursteilnehmern zu Österreich, der Schweiz oder auch darüber hinaus konfrontiert werden. Zudem ist bei der Lehrsituation im zielsprachenfernen Kontext viel weniger als bspw. im DaZ-Kontext klar, mit welchem Land die Deutschlerner in Zukunft vielleicht in Kontakt treten werden. Eine ausreichende Sensibilisierung und Vermittlung von grundlegenden Informationen ist hier also auf alle Fälle wünschenswert, zumal die Heterogenität des deutschsprachigen Sprach- und Kulturraums bei den Deutsch- bzw. Germanistikstudierenden häufig großes Interesse weckt (vgl. z. B. SCHWEIGER 2013: 133). Wir haben es hier, verkürzt gesagt, mit einem „Mehrwert der deutschen Sprache“ zu tun, bei dem sich mit einer Sprache mehrere Länder erschließen lassen - oder wie es Shafer und Baumgartner (2017: 68) pointiert formulieren: „,mehr Deutsch fürs gleiche Geld.“

Ein weiterer Aspekt ist von Belang und sollte - sicher noch viel stärker als das bisher der Fall ist - in den Blick genommen werden: die Auseinandersetzung mit der Vielfalt der deutschen Sprache und Kultur erfolgt seit einigen Jahren stetig mehr forschungsorientiert (vgl. SCHWEIGER 2013: 134f.). Die Auseinandersetzung mit dem deutschsprachigen Kultur- und Sprachraum (im weiten Sinn des Wortes) ermöglicht eine bewusste Auseinandersetzung mit kulturellen Lernprozessen und erfüllt damit eine Grundforderung an ein angemessenes, nachhaltiges Lehramtsstudium. Dass dies nicht irgendwo geschieht, sondern in Brasilien, hat eine zusätzliche Bedeutung: Aufgrund der starken deutschsprachigen ${ }^{26}$ Einwanderung seit Beginn des 19. Jahrhunderts sind Teile des Landes von der deutschen Sprache und Kultur geprägt worden, und die Herkunft der Vorfahren spielt für die Identität (und nicht zuletzt für die Sprachenwahl) nach wie vor eine wichtige Rolle. Für eine akademische Auseinandersetzung mit dem deutschen Sprachraum - gestern, heute und in Zukunft - ist also die solide Kenntnis der Vielfalt und der Dynamiken von Sprache und Kultur eine grundlegende Kompetenz, die in den kommenden Jahren sicher auch relevanter wird. „Deutsch“ eben nicht als „deutschländisch“ zu verstehen, sondern als „deutschsprachig“ ist ein genuines Ziel des DACH-Prinzips, und für Brasilien mindestens ebenso relevant wie für andere Weltregionen.

\footnotetext{
${ }^{26}$ Der Begriff „,deutschsprachig“ (und nicht „deutsch“) ist an dieser Stelle sehr bewusst gewählt. Zum einen kann vor 1871 nicht von Deutschland als „Staat“" gesprochen werden, zum anderen erfolgte die Migration tatsächlich aus ganz Europa (mit Sprechern des Deutschen als Erst- oder Zweitsprache), so z. B. aus der Schweiz (vgl. DEWULF 2007).
}

Pandaemonium, São Paulo, v. 23, n. 41, set.-dez. 2020, p. 24-50 
Und Liechtenstein? Das Land ist neben den drei „Haupt-DACH-Ländern“ zwar das einzige mit ausschließlicher Verwendung von Deutsch als Amtssprache, wird jedoch aufgrund seines geringen Einflusses auf die deutsche Sprache nicht als „Vollzentrum“, sondern als „Halbzentrum“ gewertet (vgl. AMMON 2016). Bei einer Einwohnerzahl von ca. 38.000 Menschen ${ }^{27}$ - und immerhin drei Hochschulen - ist es für Deutschlernende zwar interessant, etwas über Liechtenstein zu erfahren, das Land nimmt jedoch in der internationalen Wahrnehmung eine ganz andere Rolle ein als Deutschland, Österreich und die Schweiz. Es wird deswegen zwar in der Lehrwerkanalyse mit beachtet, aber darüber hinaus nicht weiter thematisiert und auch nicht als genuines DACH-Land behandelt.

Überhaupt erscheint es wichtig, sich argumentativ von einem Anspruch auf Vollständigkeit zu verabschieden - dieser kann heutzutage, im Zeitalter der schnellen Abrufbarkeit enzyklopädischer Daten per Internet, sowieso kaum aufrecht erhalten werden. Den Autorinnen und Autoren ging es bei der Ausformulierung des DACH-Prinzips eben nicht darum, im Fremdsprachenunterricht einen quantitativ gleichwertigen Anteil der deutschsprachigen Länder zu erreichen, sondern um die grundsätzliche Anerkennung eines vielfältigen Sprach- und Kulturraumes (vgl. DEMMIG; HÄGI; SCHWEIGER 2013: 69, SHAFER; BAUMGARTNER 2017: 67).

Daran anknüpfend ein Argument ganz praktischer Natur: Deutsch wird nicht nur in den DACH-Ländern verwendet, sondern als Amts- oder Umgangssprache auch in den Halb- oder Viertelzentren, beispielsweise in Italien (Südtirol) und manchen Regionen Rumäniens und Brasiliens. Für allgemeinsprachliche Lehrwerke ist es schlicht nicht umsetzbar, alle diese Regionen angemessen zu berücksichtigen - was nicht bedeutet, dass ein regional entwickeltes Lehrbuch bspw. für Brasilien die Gegebenheiten nicht stärker berücksichtigen könnte. Solange im Unterricht auf allgemeinsprachliche Lehrwerke zurückgegriffen wird, liegt es - gerade auch im Kontext eines Landes mit Deutsch als Minderheitensprache - vornehmlich an der Lehrkraft, den Lernern die Mehrsprachigkeitssituation bewusst zu machen und ihnen - durchaus auch in der bewussten Auseinandersetzung mit der Sprachvarietät Brasiliens - Beispiele für die Verwendung aufzuzeigen.

Es bleibt festzuhalten: Durch den Einbezug von Österreich, der Schweiz und (teilweise) Liechtenstein in die Lehrbücher wird die Forderung nach einer Berücksichtigung der DACH-

\footnotetext{
${ }^{27}$ Eurostat. Abrufbar unter: https://ec.europa.eu/eurostat/en/web/population-demography-migration-projections/ statistics-illustrated [Zugriff: 24.03.2019]

Pandaemonium, São Paulo, v. 23, n. 41, set.-dez. 2020, p. 24-50
} 
Länder erfüllt - wobei die numerische Präsenz nicht bedeutet, dass ein realistisches Bild des jeweiligen Landes gezeichnet wird. Die Darstellung von Österreich bzw. der Schweiz in DaFLehrwerken nach qualitativen Gesichtspunkten zu untersuchen wäre eine spannende und durchaus relevante Aufgabe für die Zukunft. Mindestens ebenso aufschlussreich wäre zudem ein Vergleich mit anderen Lehrmaterialien, um zu schauen, ob es Alternativen gibt, welche die DACH-Perspektive stärker berücksichtigen als die in den brasilianischen Deutschstudiengängen aktuell genutzten Lehrwerke - erfolgen könnte dies etwa aus diachroner Perspektive, mit Fokus auf regional entwickelte Ausgaben oder Materialien mit einem expliziten DACHBezug. All dies kann aus Platzgründen an dieser Stelle nicht weiter vertieft werden, ein kurzer Ausblick auf mögliche Alternativen im folgenden Teilkapitel muss hier genügen.

\section{Zusammenfassung und Fazit}

Zu Beginn dieses Artikels wurde gefragt, ob in den aktuell in Brasilien für die Lehrerausbildung genutzten, in Deutschland entwickelten Lehrwerken der DACH-Aspekt zu kurz kommt. Diese Frage wurde anhand der Ergebnisse der Analyse zunächst mit „nein“ beantwortet, und bei der genaueren Betrachtung der Lehrwerke wurde deutlich, dass in ihnen durchaus Potenzial steckt. In Hinblick auf die Darstellung der DACH-Länder versuchen die Lehrwerke, eine Ausgewogenheit zu erreichen, und DACH-Aspekte kommen auf diese Weise nicht zu kurz ${ }^{28}$ (vgl. MARQUES-SchäFER; BOLACIO; STANKE 2016: 582f.). Die im Eingangskapitel erwähnte Kritik von Lehrkräften, die eine unangemessene Gewichtung der DACH-Länder in Lehrwerken kritisieren und sich mehr Material wünschen, lässt sich damit nicht belegen. Vielmehr kann man festhalten: weniger das Fehlen von Lehrmaterialien als vielmehr die Unsicherheit im Umgang mit kulturellen Themen sorgt in der Praxis des Fremdsprachenunterrichts für Unbehagen (vgl. VOERKEL 2020: 204):

Unsicherheit bei den Lehrpersonen: Das sehr allgemein und kontextunspezifisch formulierte DACH-Prinzip lasse auch Praktiker/innen oft orientierungslos, was die 'richtige' Umsetzung betrifft, und stiesse damit auf Vorbehalte. So werde das Thema als politisch korrektes 'Mehr' und 'Muss' bei eh schon knapper Unterrichtszeit wahrgenommen - und damit als Überforderung. Die Wahrnehmung von DACH als politisch erzwungene inhaltliche Überfrachtung des Unterrichts dürfte u. a. daran liegen, dass das Thema oft als nationenzentrierte, additive Faktenhuberei verstanden sowie in Lehr- und Lernmaterialien

\footnotetext{
${ }^{28}$ Diese Aussage gilt zumindest für eine quantitative Einschätzung - inwieweit das auch für die Qualität der Darstellung gilt, dazu lohnen sich weitere Überlegungen bzw. auch empirische Studien (vgl. RÖSLER; SCHART 2016: 490f.).
}

Pandaemonium, São Paulo, v. 23, n. 41, set.-dez. 2020, p. 24-50 
bisweilen auf eine klischeehafte und z. T. fehlerhafte Überbetonung von Unterschieden zwischen den Ländern reduziert wird. (SHAFER; BAUMGARTNER 2019: 103)

Zweifelsohne lassen sich die Lehr- und Lernmaterialien für den Deutschunterricht in den nächsten Jahren nicht nur in Hinblick auf die Quantität, sondern auch auf die Qualität der Darstellung weiter verbessern. Die im Zitat erwähnte Unsicherheit rührt jedoch vor allem daher, dass das DACH-Prinzip in der Aus-, Fort- und Weiterbildung von Deutschlehrkräften bisher zu wenig berücksichtigt und somit als ein zentrales Desiderat gesehen wird (vgl. SCHWEIGER 2013: 138, SHAFER; BAUMGARTNER 2019: 112). Das verdeutlicht, wie wichtig es ist, die Ausbildung der Deutschlehrkräfte noch stärker ins Auge zu fassen, um sowohl einen Grundkonsens zur Relevanz des Themas als auch die nötigen Vorkenntnisse zur Vermittlung kultureller Themen zu erreichen (vgl. RÖSLER 2012: 218, BOHUNOVSKY 2014: 115, VOERKEL 2015, KOREIK 2018: 38ff., LIMBERGER; VON MÜHLEN 2019: 351).

Die Forderung, dem gesamten amtlich deutschsprachigen Raum in seiner Mehrdimensionalität Rechnung zu tragen, ist dabei nicht neu, sondern wird seit Anfang der 1990er Jahre immer wieder vertreten (vgl. SHAFER; BAUMGARTNER 2019: 99). Denn schon lange ist klar: Studierende sind zukünftige Multiplikatoren der deutschen Sprache und Kultur. Wenn sie also während ihres Studiums bereits mit den Grundlagen des deutschsprachigen Raums vertraut gemacht werden, steigt die Wahrscheinlichkeit, dass sie im eigenen Unterricht auch auf Aspekte der sprachlichen und kulturellen Vielfalt eingehen. Dass dies mit eigenem Erleben und einer bewussten Auseinandersetzung mit dem DACH-Raum kombiniert werden sollte, um einen nachhaltigen Effekt zu erzielen, wird dabei seit Jahren betont (vgl. z. B. SCHWEIGER 2013: 137f.).

Ein weiterer Grund, warum DACH-Themen in der Lehrerausbildung eine wichtigere Rolle zu wünschen wäre, ist der Anspruch, dass zukünftige Lehrende mit Lehrwerken und Lehrwerkanalyse vertraut sein sollten, um das zur Verfügung stehende Lehrmaterial möglichst gut nutzen zu können. Die bewusste Auseinandersetzung mit den entsprechenden Materialien verspricht über die rein inhaltliche Komponente hinaus weitere wichtige Impulse in der Ausund Weiterbildung von Lehrkräften, indem über Reflexion u. a. der kritische Blick auf Materialien und ihre Kombinationsmöglichkeiten geschult wird (vgl. LÜTGE 2016: 458, SHAFER; BAUMGARTNER 2017: 68).

Inwieweit sich darüber hinaus regionale Ansätze sowie Fort- und Ausbildungskonzepte bewähren, so wie u. a. vorgeschlagen von Voerkel (2020: 209ff), wird sich erst in Zukunft zeigen. Wichtig erscheint auf alle Fälle, kulturelle Fragestellungen (noch) stärker auf Grundlage 
empirischer Erkenntnisse zu entwickeln und darüber hinaus in sinnvolle, lernerorientierte Ausbildungskonzepte zu investieren (vgl. SCHWEIGER 2013: 138). Wenn also nur die Hälfte der brasilianischen Universitäten in ihren Deutschstudiengängen überhaupt Lehrveranstaltungen zu Landeskunde bzw. Kultur im Curriculum verankert haben, stellt das Studierende und Dozenten vor ernsthafte Schwierigkeiten, die sprachliche und kulturelle Vielfalt des deutschsprachigen Raumes im wünschenswerten Umfang zu berücksichtigen.

Bis hierzu passende Antworten und Lösungen gefunden werden, gilt es, dem gefühlten Bedarf an einer stärkeren Berücksichtigung der Vielfalt der deutschen Sprache und Kultur nachzukommen und nach Alternativen zu suchen, um die Plurizentrik und das DACH-Prinzip angemessen $\mathrm{zu}$ berücksichtigen. Möglich ist zum Beispiel die Nutzung spezifischer Zusatzmaterialien, mit denen die Standard-Lehrwerke z. T. sinnvoll ergänzt werden können Beispiele dafür wären die Publikationen von Altmayer (2016), Faigle (2006), Matecki (2000) oder auch Wehage $(2018)^{29}$. Interessant sind in diesem Zusammenhang möglicherweise auch Materialien für die „Integrationskurse“, die zwar einen ganz bestimmten Zweck verfolgen und im DaZ-Kontext verortet sind, sich aber in den letzten Jahren auch professionalisiert haben. Mit diesen Angeboten könnte deswegen - durchaus in einer kritischen Perspektive - auch in der Lehrerausbildung sinnvoll gearbeitet werden; ein Beispiel dafür stellt Butler et al. (2017) dar. Mit dem Bewusstsein für mögliche Lücken in den aktuell genutzten Lehrwerken und dem Wissen über mögliche Alternativen und Ergänzungen ist es somit zweifelsohne möglich, das DACH-Prinzip auch in Brasilien adäquat und nachhaltig zu vermitteln.

\section{Literaturverzeichnis}

Auswärtiges AMT. Deutsch als Fremdprache weltweit. Datenerhebung 2015. Abrufbar unter: www.goethe.de/resources/files/pdf37/Bro_Deutschlernerhebung_final2.pdf [Zugriff am 02.02.2020].

Ahrenholz, Bernt. Deutsch als Fremdsprache - Deutsch als Zweitsprache. Orientierungen. In: OOMEN-WelKe, Ingelore; AHRENHOlZ, Bernt (Hg.). Deutsch als Fremdsprache. Baltmannsweiler: Schneider Verlag Hohengehren, 2017, p. 3-10.

AMmon, Ulrich et al. Variantenwörterbuch des Deutschen. Die Standardsprache in Österreich, der Schweiz, Deutschland, Liechtenstein, Luxemburg, Ostbelgien und Südtirol sowie Rumänien, Namibia und Mennonitensiedlungen. Berlin: De Gruyter, 2016.

ANDRADE E SILVA, Mariana. Materiais didáticos no ensino de Alemão como Língua Estrangeira para o público universitário. In: ABEG (Hg.). Anais do $1^{\circ}$ Congresso da Associação Brasileira de Estudos Germanísticos (ABEG). São Paulo: USP, 2015, p. 224-232.

\footnotetext{
${ }^{29}$ Für die sprachliche Seite finden sich Hinweise u. a. bei Bohunovsky (2014: 113f.).

Pandaemonium, São Paulo, v. 23, n. 41, set.-dez. 2020, p. 24-50
} 
VOERKEL, J.P. ${ }_{j}$ HUANG, Z. - Plurizentrik und DACH-Bezug bei Lehrwerken

BoHUNOVSKY, Ruth. Paradeiser também são tomates? - Sobre o alemão austríaco no ensinoaprendizagem de alemão no Brasil. In: MouRA, Magali et al (Hg.). Ensino-aprendizagem de alemão como língua estrangeira: teoria e práxis. Rio de Janeiro: Letra Capital, 2014, p. 110125.

BOlacio, Ebal; Voerkel, Paul; STANKe, Roberta. Material didático em cursos de alemão no contexto acadêmico brasileiro - entre conceitos gerais e específicos. In: MouRA, Magali et al. (Hg.). Ensino-aprendizagem de alemão como língua estrangeira: teoria e práxis. Rio de Janeiro: Dialogarts, 2017, p. 15-37.

CHEN, Eva. A falta de material. In: FunK, Hermann; BolaCiO, Ebal (Hg.). Kulturdidaktik im Unterricht Deutsch als Fremdsprache. Rio de Janeiro: APA-Rio, 2014, p. 42-65.

DeMmig, Silvia; HÄGI, Sara; SCHWEIGER, Hannes (Hg.). DACH-Landeskunde: Theorie - Geschichte Praxis. München: Iudicium, 2013.

DEWULF, Jeroen. Brasilien mit Brüchen. Schweizer unter dem Kreuz des Südens. Zürich: Verlag Neue Zürcher Zeitung, 2007.

FORNOFF, Roger. Landeskunde und kulturwissenschaftliche Gedächtnisforschung. Erinnerungsorte des Nationalsozialismus im Unterricht Deutsch als Fremdsprache. Baltmannsweiler: Schneider Verlag Hohengehren, 2016.

FUNK, Hermann. Qualitätsmerkmale von Lehrwerken prüfen - ein Verfahrensvorschlag. In: Babylonia 3/04, 2004, p. 41-47. Abrufbar unter: http://babylonia.ch/fileadmin/user_upload/ documents/2004-3/funk.pdf [Zugriff am 22.02.2019].

FUNK, Hermann. Lehrwerkforschung. In: HALLET, Wolfgang; KÖNIGS, Frank (Hg.). Handbuch Fremdsprachendidaktik. Seelze: Klett Kallmeyer, 2010, p. 364-368.

FUNK, Hermann. Lehrwerkanalyse als Ausbildungsinhalt in der Ausbildung von Lehrpersonal. In: FUNK, Hermann; BOLACIO, Ebal (Hg.). Kulturdidaktik im Unterricht Deutsch als Fremdsprache. Rio de Janeiro: APA-Rio, 2014, p. 66-87.

FunK, Hermann. Lehr-/ Lernmaterialien und Medien. In: BurwitZ-Melzer, Eva et al. (Hg.). Handbuch Fremdsprachenunterricht. Tübingen: A. Francke, 2016, p. 435-441.

GONDAR, Anelise; FerReIRA, Meg. Desafios do uso do livro didático no ensino superior: uma análise empírica exploratória da recepção de aspectos de vocabulário, gramática e exercícios do livro DaF kompakt. Pandaemonium Germanicum, São Paulo, v. 22, n. 37, p. 302-330, 2019.

HÄGI, Sara. Aber bitte richtig mit Sahne, Rahm oder Schlag(obers): Voraussetzungen für eine adäquate Umsetzung des plurizentrischen Ansatzes im DaF-Unterricht. In: ABEL, Andrea; STUFLESSER, Mathias; PUTZ, Magdalena (Hg.). Mehrsprachigkeit in Europa. Erfahrungen, Bedürfnisse, Gute Praxis. Bozen/Bolzano: Eurac, 2006, p. 273-284.

IDV (= Der Internationale Deutschlehrerverband). ABCD-Thesen zur Rolle der Landeskunde im Deutschunterricht. In: IDV-Rundbrief 45 (September 1990), p. 15-18.

JARZĄBEK, Alina Dorota. Der plurizentrische Ansatz und das DACH-Prinzip in polnischen DaFLehrwerken. Germanica Wratislaviensia 138, 2013, p. 173-183.

KAST, Bernd; NEUNER, Gerhard (Hg.). Zur Analyse, Begutachtung und Entwicklung von Lehrwerken für den fremdsprachlichen Deutschunterricht. Berlin: Langenscheidt, 1994.

KOREIK, Uwe. Das ,deutsche Wirtschaftswunder‘. Mythos, Legende oder ein Erinnerungsort - die Relevanz für Deutsch als Fremd- und Zweitsprache? In: SCHIEDERMAIR, Eva Simone (Hg.). Deutsch als Fremd- und Zweitsprache und Kulturwissenschaft. Zugänge zu sozialen Wirklichkeiten. München: Iudicium, 2018, p. 27-46.

LIMBERGER, Bernardo; VON MÜHLEN, Fernanda. Variação linguística e alemão como língua estrangeira: contribuições a partir da análise de dois livros didáticos. Pandaemonium Germanicum, São Paulo, v. 22, n. 37, p. 331-356, 2019.

LÜTGE, Christiane. Lehr-/Lernmaterialien und Medien zum Aufbau interkultureller Kompetenzen. In: BurWITZ-MElZER, Eva et al. (Hg.). Handbuch Fremdsprachenunterricht. Tübingen: A. Francke, 2016, p. $456-459$.

Pandaemonium, São Paulo, v. 23, n. 41, set.-dez. 2020, p. 24-50 
VOERKEL, J.P. $;$ HUANG, Z. - Plurizentrik und DACH-Bezus bei Lehrwerken

MARQUES-SCHÄFER, Gabriela, BOLACIO, Ebal; STANKE, Roberta Sol. Was können Lehrwerke zur Reflexion von Stereotypen im DaF-Unterricht beitragen? Eine Analyse anhand der Arbeit mit DaF kompakt in Brasilien. InfoDaF, Heft 5/2016, p. 566-586.

MuHR, Rudolf. Das Österreichische Sprachdiplom Deutsch. Lernzielkatalog. Wien: ÖBV, 2000.

NIEWELER, Andreas. Lehrwerk. In: SURKAMP, Carola (Hg.). Metzler Lexikon Fremdsprachendidaktik. Ansätze - Methoden - Grundbegriffe. Stuttgart: Metzler, 2017a, p. 206-208.

NIEWELER, Andreas. Lehrwerkanalyse. In: SURKAMP, Carola (Hg.). Metzler Lexikon Fremdsprachendidaktik. Ansätze - Methoden - Grundbegriffe. Stuttgart: Metzler, 2017b, p. 208-209.

RÖSLER, Dietmar. Deutsch als Fremdsprache. Eine Einführung. Stuttgart: Metzler, 2012.

RÖSLER, Dietmar; SCHART, Michael. Die Perspektivenvielfalt der Lehrwerkanalyse - und ihr weißer Fleck. InfoDaF, n. 5, p. 483-493, 2016.

RÖSLER, Dietmar; WÜRFFEL, Nicola. Lernmaterialien und Medien. München: Langenscheidt, 2014. [Materialien DLL, Band 5].

SCHMIDT, Cristiane. Estudo do livro didático de língua alemã: abordagem sociocomunicativa e intercultural. [Tese de doutorado em Letras, Universidade Estadual do Oeste do Paraná, 2016].

SCHWEIGER, Hannes. DACH erleben. Das DACH-Konzept in der Fortbildung von Lehrenden. In: DEMMIG, Silvia; HÄGI, Sara; SCHWEIGER, Hannes (Hg.). DACH-Landeskunde: Theorie Geschichte - Praxis. München: Iudicium, 2013, p. 129-140.

SHAFER, Naomi; BAUMgARTNER, Martin. Mehr als Länder- oder Landeskunde: Ansätze eines weitergedachten DACH-Prinzips. In: INTERNATIONALER DEUTSCHLEHRERVERBAND - IDV (Hg.). IDV-Magazin, n. 92, p. 67-71, 2017.

Shafer, Naomi; BAuMgartner, Martin. Die Pluralität von DaF als Plus: Zu einem weitergedachten DACH-Prinzip. In: FORSTER Vosicki, Brigitte; GICK, Cornelia; STUDER, Thomas (Hg.). Sprachenpolitik: Expertenberichte und Freiburger Resolution. [Tagungsband 3 der IDT 2017]. Berlin: Erich Schmidt, 2019, p. 98-114.

SILVEIRA, Raphael. O livro didático nas aulas de alemão como língua estrangeira na universidade: reflexões a partir de uma sondagem. In: ABEG (Hg.). Anais do $1^{\circ}$ Congresso da Associação Brasileira de Estudos Germanísticos (ABEG). São Paulo: USP, 2015, p. 275-283.

VOERKEL, Paul. Der Landeskunde auf der Spur: Zur Entwicklung von Kulturkonzeptionen in der DaFLandeskunde. In: MourA, Magali et al. (Hg.). Ensino-aprendizagem de alemão como língua estrangeira: teoria e práxis. Rio de Janeiro: Letra Capital, 2015, p. 91-110.

VOERKEL, Paul. Deutsch als Chance: Ausbildung, Qualifikation und Verbleib von Absolventen brasilianischer Deutschstudiengänge. [Dissertationsarbeit an der Friedrich-Schiller-Universität Jena, 2017]. Verfügbar unter: https://www.db-thueringen.de/receive/dbt_mods_00033644 [Zugriff: 31.10.2019].

VoerKel, Paul. Das DACH-Prinzip im zielsprachenfernen Kontext. In: SHAFER, Naomi et al. (Hg.). Weitergedacht. Das DACH-Prinzip in der Praxis. Göttingen: Universitätsverlag, 2020, p. 195216.

\section{Genannte Lehrwerke}

Albrecht, Ulrike et al. (Hg.). Passwort Deutsch. Stuttgart: Klett, 2005.

AltMAYer, Claus et al. (Hg.). Mitreden. Diskursive Landeskunde für Deutsch als Fremd- und Zweitsprache. Stuttgart: Klett, 2016.

Braun, Birgit et al. (Hg.). DaF Kompakt neu. Stuttgart: Klett, 2016.

Braun, Birgit et al. (Hg.). DaF Kompakt. Stuttgart: Klett, 2011.

Buscha, Anne; RAVEN, Susanne; SzITA, Szilvia. Erkundungen. Leipzig: Schubert, 2014.

Pandaemonium, São Paulo, v. 23, n. 41, set.-dez. 2020, p. 24-50 
BuTLER, Ellen et al. (Hg.). 100 Stunden Deutschland. Orientierungskurs Politik - Geschichte - Kultur. Stuttgart: Klett, 2017.

DallaPiAZZA, Rosa-Maria et al. (Hg). Ziel. München: Hueber, 2009.

DANIELS, Albert et al. (Hg.). Mittelpunkt. Stuttgart: Klett, 2007.

FAIGLE, Iris. Rundum. Einblicke in die deutschsprachige Kultur. Stuttgart: Klett, 2006.

FunK, Hermann et al. (Hg). Studio d. Berlin: Cornelsen, 2005.

FunK, Hermann et al. (Hg). Studio 21. Berlin: Cornelsen, 2015.

Glas-Peters, Sabine; Pude, Angela; ReIMAnN, Monika. Menschen. München: Hueber, 2015.

JeNTGES, Sabine et al. (Hg.). Aussichten. Stuttgart: Klett, 2009.

KoITHAN, Ute et al. (Hg.). Aspekte. Stuttgart: Klett, 2013.

Krenn, Wilfried; PuCHTA, Herbert. Motive. München: Hueber, 2014.

LEMCKE, Christiane et al. (Hg.). Optimal. München: Langenscheidt, 2004.

MATECKI, Uta. Dreimal Deutsch.. Stuttgart: Klett, 2000.

MoELLER, Jack et al. (Hg.). Deutsch heute. Introductory German. Boston: Cengage, 2010.

PenNing-Hiemstra, Sylvette et al. (Hg.). Schritte. München: Hueber, 2005.

Perlmann-Balme, Michaela et al. (Hg.). Em. München: Hueber, 1998.

WeHAGE, Franz-Joseph. DACHL. Unterwegs in deutschsprachigen Ländern. A Cultural Reader \& Workbook for Advanced Intermediate German \& Beyond. Indianapolis: Hackett, 2018. 